\title{
Prevalence and sex- and age-related risk of pulmonary embolism in in-hospital patients with atrial fibrillation: a multicenter retrospective study from China
}

\author{
Ying Bai ${ }^{1}$, Qiao-Min Yue ${ }^{1}$, He Sun ${ }^{1}$, Shi-Dong Guo ${ }^{2}$, Zhen-Zhou Wang ${ }^{3}$, Peng Zhong ${ }^{4}$, Xue-Yao Wei ${ }^{5}$, \\ Lin Sun ${ }^{6}$, Yue Liu ${ }^{7}, \mathrm{Xu}-\mathrm{Bo} \mathrm{Shi}^{1}$, Yan-Ting Liu ${ }^{5}$ \\ ${ }^{1}$ Cardiovascular Center, Beijing Tongren Hospital, Capital Medical University, Beijing, China; ${ }^{2}$ Emergency Department, China-Japan Friendship \\ Hospital, Beijing, China; ${ }^{3}$ Trauma Medicine Center, Peking University People’s Hospital, China; ${ }^{4}$ Department of Ultrasound, Beijing Friendship \\ Hospital, Capital Medical University, Beijing, China; ${ }^{5}$ Information Center, Beijing Tongren Hospital, Capital Medical University, Beijing, China; \\ ${ }^{6}$ Neurologic Department, Beijing Tongren Hospital, Capital Medical University, Beijing, China; ${ }^{7}$ Department of Cardiology, The First Affiliated \\ Hospital of Harbin Medical University, Harbin, China \\ Contributions: (I) Conception and design: Y Bai; (II) Administrative support: XB Shi, YT Liu, SD Guo, ZZ Wang; (III) Provision of study materials \\ or patients: YT Liu, SD Guo, P Zhong, ZZ Wang, XY Wei; (IV) Collection and assembly of data: Y Bai, QM Yue, H Sun, L Sun, Y Liu, XB Shi; \\ (V) Data analysis and interpretation: Y Bai, QM Yue, H Sun, L Sun, Y Liu, XB Shi; (VI) Manuscript writing: All authors; (VII) Final approval of \\ manuscript: All authors. \\ Correspondence to: Ying Bai. Cardiovascular Center, Beijing Tongren Hospital, Capital Medical University, No.1, Dong Jiao Min Xiang Street, \\ Dongcheng District, Beijing 100730, China. Email: byfeixue@sina.com; Yan-Ting Liu. Information Center, Beijing Tongren Hospital, Capital \\ Medical University, No. 1, Dong Jiao Min Xiang Street, Dongcheng District, Beijing 100730, China. Email: bjtrliu@126.com.
}

\begin{abstract}
Background: This study was designed to explore the prevalence of pulmonary embolism (PE) and sex and age-related risk of incident $\mathrm{PE}$ in in-hospital patients with atrial fibrillation (AF) in China.

Methods: A retrospective cohort of 15,688 AF patients (mean age: 72.56 years; $55.7 \%$ male) was identified from 2008 to 2018 in our hospitals. The prevalence and incidence of PE over a 2.28-year follow-up were studied. Unadjusted, age or sex-adjusted, and multivariate Cox regression were used to explore the risk of PE in the studied patients.

Results: One hundred eighty-two AF patients (1.2\%) had PE at their first hospitalizations. Over a mean follow-up of 2.28 years, 85 patients developed PE, with an incidence of $0.24 \%$ per person-year. PE was more likely to occur in female and older patients with AF according to the unadjusted, age or sex-adjusted, and multivariate Cox regression analysis (all $\mathrm{P}<0.05)$. Moreover, a significant higher risk of $\mathrm{PE}$ was seen in female and older patients in AF using Kaplan-Meier analysis, respectively (log-rank: both $\mathrm{P}<0.001$ ).

Conclusions: In the current AF cohort, the prevalence of PE was $1.2 \%$ and the incidence of $\mathrm{PE}$ was $0.24 \%$ per person-year during a mean follow-up of 2.28 years. Female and older patients were more likely to experience PE compared to male and younger patients.
\end{abstract}

Keywords: Atrial fibrillation (AF); pulmonary embolism (PE); incidence; female; older patients

Submitted Mar 20, 2020. Accepted for publication Aug 21, 2020.

doi: $10.21037 /$ atm-20-2718

View this article at: http://dx.doi.org/10.21037/atm-20-2718

\section{Introduction}

Pulmonary embolism (PE) is a potentially life-threatening condition and generally thought to be related to the peripheral deep vein thrombosis (DVT) (1). However,
DVT was not revealed in all PE patients by ultrasound or other imaging techniques. Therefore, other sources of thrombus were investigated, especially when the patients were complicated with conditions associated with clot 
formation, such as atrial fibrillation (AF) (2). The overlap of $\mathrm{PE}$ and $\mathrm{AF}$ was noted for decades with the common concept of stagnant blood flow in the right atrium (RA) during AF leading to thrombus formation in RA and subsequent PE (3).

Many factors were suggested to increase the risk of $\mathrm{PE}$, such as heart failure, active malignancy, etc. A previous study showed higher incidence of PE in female and older patients (4) and these patients also had a higher risk of systematic embolism when complicated with $\mathrm{AF}$ (5). As the mechanism of systematic embolism and $\mathrm{PE}$ were similar in $\mathrm{AF}$ patients with thrombus arising from the left or right atria, we speculated that among AF patients, female and older patients might experience higher risk of $\mathrm{PE}$ as well. Thus, this study was designed to investigate the incidence of $\mathrm{PE}$ according to age and sex differences in an in-hospital AF cohort from China.

We present the following article in accordance with the STROBE reporting checklist (available at http://dx.doi. org/10.21037/atm-20-2718).

\section{Methods}

\section{Study patients}

Hospitalization data from three hospitals was used for analysis, including Beijing Tongren Hospital, Capital Medical University (from September 2008 to December 2018), Beijing Friendship Hospital, Capital Medical University (from January 2013 to March 2018) and ChinaJapan Friendship Hospital (from April 2011 to May 2018). All these hospitals were tertiary hospitals in Beijing, China. Initially, 11,259, 9,579 and 5,968 visits of AF patients were respectively extracted from the database of each hospital. After excluding the repeated admissions, 6,668 first admissions from Beijing Tongren Hospital, 5,762 from Beijing Friendship Hospital and 3,258 from China-Japan Friendship Hospital were included in the present study.

The study was conducted in accordance with the Declaration of Helsinki (as revised in 2013). The study was approved by clinical research ethics committee of our centers (NO.: TRECKY2020-097) and individual consent for this retrospective analysis was waived.

\section{Data collection}

Patients' diagnoses documented as the International Classification of Disease-10th edition (ICD-10) codes, including ICD 48 (I48xx1, I48xx02, I48xx03, $\mathrm{I} 48 \mathrm{xx} 04, \mathrm{I} 48 \mathrm{xx} 05, \mathrm{I} 48 \mathrm{xx} 06, \mathrm{I} 48 \mathrm{xx} 07, \mathrm{I} 48 \mathrm{xx} 08$,
I48xx09, I48xx10, I48xx11, I48xx12, I48xx13, I48xx14), were collected. The patients' information was obtained from the database, including demographics (e.g., age, sex), comorbidities and discharge status. Data on echocardiography examinations was obtained as well and left atrial (LA) diameter was assessed in the present study.

Primary diagnoses were collected from records of outpatient visits or inpatient readmissions by searching health insurance numbers or case numbers after first discharges from the three hospitals until 2018.

\section{$P E$}

Patients' records with the diagnosis of $\mathrm{PE}$ were then obtained for more detailed information. The event of $\mathrm{PE}$ was confirmed if anticoagulation treatment was performed due to the diagnosis combining symptoms, clinical signs, D-dimer, blood gas test and objective examinations, such as pulmonary artery computed tomography angiography (CTA) and/or perfusion-ventilation (V/Q) lung scan.

Patients with the primary diagnoses of $\mathrm{PE}$ were subsequently identified whether $\mathrm{PE}$ was previously existed or new-onset. And the latter was further defined as prevalent $\mathrm{PE}$ if it occurred during the first hospitalizations and as incident $\mathrm{PE}$ if it occurred after discharges. Incident $\mathrm{PE}$ was considered as the termination event.

\section{Statistics}

Continuous variables were expressed as mean \pm SD and compared by unpaired Student's $t$-tests. Categorical variables were expressed as number (percentage \%) and compared using Chi-square tests. The prevalence of $\mathrm{PE}$ in the AF cohort was calculated while the incidence of $\mathrm{PE}$ was shown as number and person-year and expressed as the event rate. The differences between sex-specific and age-categorized $\mathrm{PE}$ incidence in $\mathrm{AF}$ were analyzed by Chi-square tests. Unadjusted and adjusted sex or agerelated risk of incident $\mathrm{PE}$ were obtained by univariate and multivariate Cox regression with a stepwise method. Factors in the multivariate analysis included sex, age, AF type, hypertension, diabetes mellitus, dyslipidemia, heart failure history, baseline myocardial infarction, peripheral artery disease, DVT, anemia, chronic kidney disease (CKD) stage III-V, which was defined according to The National Kidney Foundation/Kidney Disease Outcome Quality Initiative (NKF/K-DOQI), stroke history and active malignancy. Kaplan-Meier analysis stratified by sex and age was then 
Table 1 Baseline characteristics of the included patients

\begin{tabular}{|c|c|c|c|c|}
\hline Variables & Total & No new onset PE & New onset PE & $P$ value \\
\hline Age & $72.56 \pm 11.65$ & $72.47 \pm 11.69$ & $77.58 \pm 8.20$ & $<0.001$ \\
\hline Female, n (\%) & $6,954(44.3)$ & $6,809(44.1)$ & $145(55.8)$ & $<0.001$ \\
\hline Paroxysmal AF, n (\%) & $7,343(46.2)$ & $7,223(60.2)$ & $120(50.2)$ & 0.001 \\
\hline Hypertension, n (\%) & $10,549(67.2)$ & $187(71.9)$ & $10,362(67.2)$ & 0.11 \\
\hline Diabetes mellitus, n (\%) & 4,892 (31.2) & $4,813(31.2)$ & $79(30.4)$ & 0.84 \\
\hline CKD III-V, n (\%) & $2,428(15.5)$ & $2,375(15.4)$ & $53(20.4)$ & 0.03 \\
\hline Prior HF, n (\%) & $5,316(33.9)$ & $5,208(33.8)$ & $108(41.5)$ & 0.009 \\
\hline Prior gastric disease, $\mathrm{n}(\%)$ & $706(4.5)$ & $690(4.5)$ & $16(6.2)$ & 0.22 \\
\hline Chronic lung disease, n (\%) & $3,069(19.6)$ & $3,004(19.5)$ & $65(25.0)$ & 0.03 \\
\hline Anemia, n (\%) & $2,078(13.2)$ & 2,036 (13.2) & $42(16.2)$ & 0.16 \\
\hline DVT, n (\%) & $533(3.4)$ & $464(3.0)$ & $69(26.5)$ & $<0.001$ \\
\hline MI, n (\%) & $2,132(13.6)$ & 2,105 (13.6) & $27(10.4)$ & 0.15 \\
\hline Active malignancy, n (\%) & $1,456(9.3)$ & $1,431(9.3)$ & $25(9.6)$ & 0.84 \\
\hline LAD, cm & $4.09 \pm 0.77$ & $4.09 \pm 0.77$ & $4.02 \pm 0.73$ & 0.32 \\
\hline LVEF, \% & $61.16 \pm 10.54$ & $61.15 \pm 10.55$ & $61.59 \pm 10.12$ & 0.66 \\
\hline
\end{tabular}

$\mathrm{PE}$, pulmonary embolism; NA, not available; AF, atrial fibrillation; CKD, chronic kidney dysfunction; HF, heart failure; TIA, transient ischemic attack; PAD, peripheral artery disease; DVT, deep vein thrombosis; MI, myocardial infarction; LAD, left atrial diameter; LVEF, left ventricular ejection fraction; LVEDD, end-diastolic left ventricular diameter.

performed using log-rank analysis. $\mathrm{P}<0.05$ was considered as statistical significance. All statistical analyses were performed with SPSS 23.0 software (SPSS Inc., Chicago, IL, USA).

\section{Results}

\section{Baseline characteristics}

Of the 15,688 AF patients (mean age: 72.56 years; $44.3 \%$ female), 260 had new-onset PE, including 182 prevalent $\mathrm{PE}$ and 85 incident $\mathrm{PE}$ (seven patients suffered both prevalent and incident $\mathrm{PE}$ ). Among patients with $\mathrm{PE}, 130$ were diagnosed by V/Q lung scan and 117 by pulmonary artery CTA. Diagnoses of the other 18 were made by combined V/Q lung scan and pulmonary artery CTA. Two of the patients were diagnosed by combining suspected symptoms, increased D-dimer and abnormal blood gas analysis. Table 1 showed the baseline characteristics of the included patients. Patients with new-onset PE were more likely to be older, female and had non-paroxysmal AF, CKD stage III-V, prior HF, prior stroke/TIA and chronic lung disease history compared to those without new-onset PE.

\section{New onset PE during first bospitalizations}

One hundred eighty-two patients (1.2\%) had prevalent PE at their first hospitalizations in the three hospitals.

\section{New-onset PE following discharge}

Eighty-five AF patients (0.24\% per person-year) 
Table 2 Risk of incident pulmonary embolism in patients with atrial fibrillation

\begin{tabular}{|c|c|c|c|c|c|c|}
\hline Model & \multicolumn{3}{|c|}{ Sex } & \multicolumn{3}{|c|}{ Age } \\
\hline Model 1 & 2.25 & $1.44-3.50$ & $<0.001$ & 1.06 & $1.04-1.09$ & $<0.001$ \\
\hline Model 2 & 2.07 & $1.32-3.22$ & 0.001 & $\mathrm{NA}$ & NA & NA \\
\hline Model 3 & NA & NA & NA & 1.06 & $1.04-1.09$ & $<0.001$ \\
\hline
\end{tabular}

Model 1, Unadjusted Cox regression; Model 2, Age adjusted Cox regression; Model 3, Sex adjusted Cox regression; Model 4, Multivariate Cox regression. Adjusted factors including sex, age, atrial fibrillation type, hypertension, diabetes mellitus, dyslipidemia, heart failure history, baseline myocardial infarction, peripheral artery disease, deep vein thrombosis, anemia, chronic kidney disease stage III-V, stroke history and active malignancy. PE, pulmonary embolism; AF, atrial fibrillation; NA, not available.

Table 3 Sex-specific and age-categorized incident pulmonary embolism in atrial fibrillation (N\% per person-year)

\begin{tabular}{lcccc}
\hline Age, year & Total & Male & Female & P value \\
\hline$<50$ & 0 & 0 & 0 & NA \\
$50-74$ & $24(0.14)$ & $7(0.07)$ & $17(0.22)$ & 0.006 \\
$\geq 75$ & $61(0.37)$ & $23(0.27)$ & $38(0.49)$ & 0.02 \\
Total & $85(0.24)$ & $30(0.15)$ & $55(0.34)$ & 0.0002 \\
\hline
\end{tabular}

N, number; NA, not available.

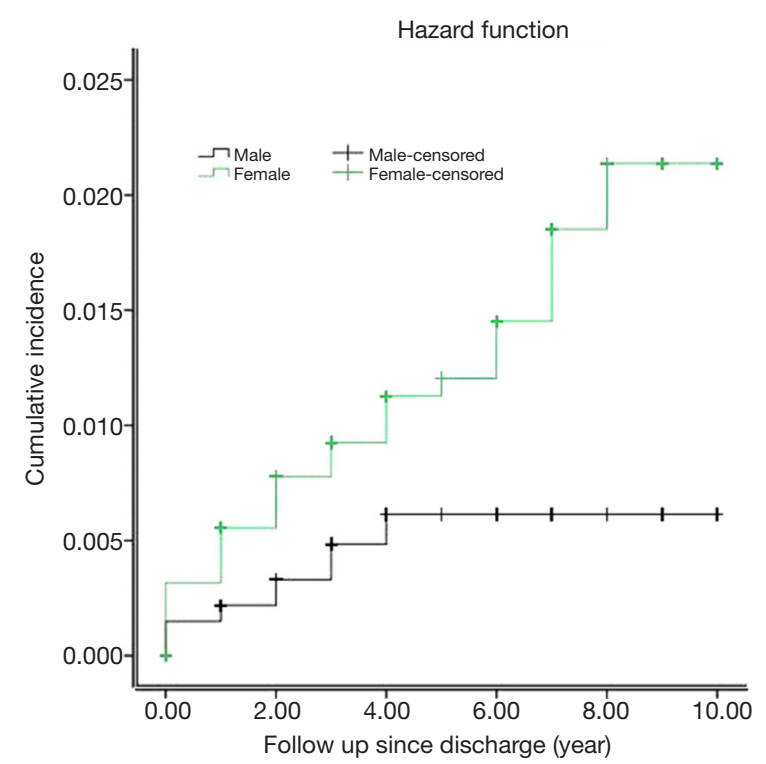

Figure 1 A higher incidence of $\mathrm{PE}$ was found in female compared with male using Kaplan-Meier analysis. (log-rank: $\mathrm{P}<0.001) . \mathrm{PE}$, pulmonary embolism. experienced incident PE during a follow-up of $2.28 \pm 2.43$ years. Female patients were shown to have higher risk of $\mathrm{PE}$ than male patients in the unadjusted, age-adjusted, and multivariate Cox regression analyses (all $\mathrm{P}<0.05$ ) (Table 2).

Furthermore, incident PE were more likely to occur in older patients rather than younger patients with $\mathrm{AF}$ according to unadjusted, sex-adjusted and multivariate Cox regression analyses. None of the patients with age less than 50 developed PE in the current cohort (Table 3). However, patients with age more than 75 years old doubled the risk of PE compared to those with age less than 75 years old (HR: 2.92, 95\% CI: 1.82-4.68).

A higher incidence of PE in female patients was found compared to male patients by Kaplan-Meier analysis (logrank: $\mathrm{P}<0.001$, Figure 1) and such increased risk of $\mathrm{PE}$ was shown in patients more than 75 years old compared to those less than 75 years old as well. (log-rank: $\mathrm{P}<0.001$, Figure 2).

\section{Discussion}

This paper reported not only the prevalence of $\mathrm{PE}$, but also the incidence of PE in AF patients in an in-hospital cohort from China. We also found a higher incidence of PE in 


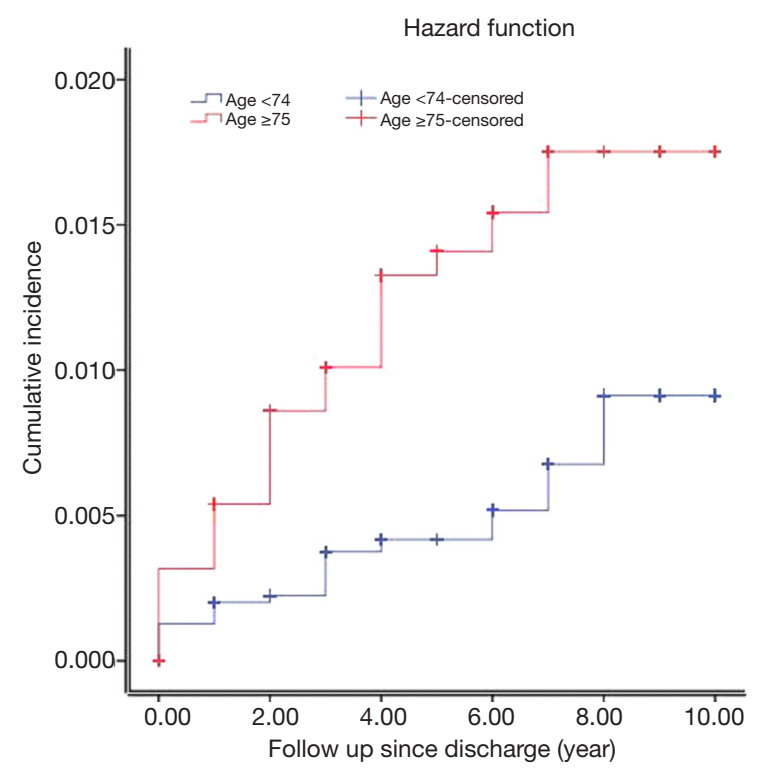

Figure 2 A higher incidence of PE was found in older patients compared with younger patients using Kaplan-Meier analysis. (logrank: $\mathrm{P}<0.001)$. $\mathrm{PE}$, pulmonary embolism.

female and older patients with $\mathrm{AF}$.

\section{Prevalence of PE in $A F$}

To our knowledge, this is the first report about the incidence of $\mathrm{PE}$ in $\mathrm{AF}$ patients in China. Keller et al. (6) found a prevalence of $0.12 \%$ of $\mathrm{PE}$ in $\mathrm{AF}$ patients in Germany, lower than the current report (1.2\%). One possible explanation was the overlap in antithrombotic therapy for $\mathrm{PE}$ and $\mathrm{AF}$ and better antithrombotic treatment would be beneficial for PE prevention in $\mathrm{AF}$ patients (7). Big gaps in anticoagulation still existed between European countries and China, though substantial improvement was seen in China in the recent years (8).

\section{Incidence of $\mathrm{PE}$ in $\mathrm{AF}$}

AF was considered as one risk factor contributing to the incident PE in a previous study (9). Furthermore, AF had a negative impact on the risk of embolism, not only during the stay in hospital, but also in the extended follow-up (10). Recently, apart from the theory associated with the clot formation in RA, several papers debated whether AF increased the risk of VTE using cohorts from communities or hospitals. Some studies supported the association between $\mathrm{AF}$ and increased risk of VTE during extended follow-up $(11,12)$. However, as the risk of DVT was independently increased by the concomitant presence of lower leg fractures, major surgeries and neoplasms, no association was found between AF and VTE formation in another study excluding these patients (13). In our report, the incidence of PE (2.4/1,000 person) was similar to the reports of Wang et al. (12) (1.5/1,000 person) and Basili et al. (13) $(0.65 / 1,000$ person), but lower than the report of Lutsey et al. (6.3/1,000 person-year) (11) when the whole in-hospital AF cohort was used for calculation.

\section{Risk of PE in female with AF}

Clot formation in RA was assumed to be one underlying mechanism of PE, similar to the cause of systematic embolism. Female was shown to have higher risk of systematic embolism and involved in the risk stratification score CHA2DS2-VASc score (5). As suspected, female was found to be associated with increased risk of PE in the current study. Stein et al. (4) showed higher risk of $\mathrm{PE}$ in women in the general population. The reported incidence of $\mathrm{PE}$ was $0.23 \%$ which was similar to our report. Actually, not just symptomatic PE but also silent PE would be included in the current study, as D-dimmer was routinely examined in our centers and then blood gas and imaging examinations (V/Q lung scan and/or pulmonary artery CTA) were performed if necessary. The relationship between sex and risk of PE was debated for decades. A previous study (14) argued that men had a higher risk while other studies $(15,16)$ argued that the risk was comparable between men and women. In our study, the age-categorized analysis showed that none of the patients with age below 50 had PE. Therefore, women with age above 50 had higher risk of PE, consistent with the study by Stein et al. (4).

\section{The possible reason was that both $\mathrm{PE}$ and $\mathrm{AF}$ were closely related with old age}

When the risk of PE was studied using age-categorized analysis, old age was shown to be related to an increased risk of PE. In the category of age less than 50 years old, no incident PE was found in the present study. Thus, we believed that more attention should be paid to female and older patients with $\mathrm{AF}$ in the clinical practice, not just for the anticoagulation treatment to prevent $\mathrm{PE}$, but also for the definite diagnosis of $\mathrm{PE}$ at the time of suspected symptoms.

\section{Limitations}

There are several limitations to be mentioned for this paper. 
First, patients usually neglected occult PE and wouldn't see doctors, which might lead to an underestimation of the incidence of PE (17). Second, potential PE cases were derived from discharge diagnosis registry and clinical records from the three hospitals. Thus, patients with PE treated in other hospitals would have been missed. However, the possibility of missing new-onset $\mathrm{PE}$ was reduced to the maximum by checking the outpatient records for possible PE diagnosis, because most inhabitants in Beijing would seek medical advice nearby. Third, the underlying diseases of the included patients were complicated, including DVT, cardiac insufficiency, lung diseases, and dependent abilities which had the probability to lead to PE. Moreover, the relationship between $\mathrm{PE}$ and $\mathrm{AF}$ was hardly confirmed using the technique of the current study. Fourth, as known, some other fatal underlying diseases, such as aortic dissection and heart failure, might have similar changes in imaging examinations, which might lead to a confounded diagnosis and then the risk of over-estimation of PE.

\section{Conclusions}

In an in-hospital AF cohort from China, the prevalence of PE was $1.2 \%$. During a mean follow-up of 2.28 years, the incidence of $\mathrm{PE}$ was $0.24 \%$ per person-year. Females and older patients were more likely to experience PE compared to male and younger patients with $\mathrm{AF}$.

\section{Acknowledgments}

Funding: This study was supported by grants from Beijing Tongren Hospital, Capital Medical University (2016-YJJBJRC-010), National Science Foundation of China (81800291), Beijing Hospitals Authority Youth Programme (QML20180207).

\section{Footnote}

Reporting Checklist: The authors have completed the STROBE reporting checklist. Available at http://dx.doi. org/10.21037/atm-20-2718

Data Sharing Statement: Available at http://dx.doi. org/10.21037/atm-20-2718

Conflicts of Interest: All authors have completed the ICMJE uniform disclosure form (available at http://dx.doi. org/10.21037/atm-20-2718). The authors have no conflicts of interest to declare.

Ethical Statement: The authors are accountable for all aspects of the work in ensuring that questions related to the accuracy or integrity of any part of the work are appropriately investigated and resolved. The study was conducted in accordance with the Declaration of Helsinki (as revised in 2013). The study was approved by clinical research ethics committee of our centers (NO.: TRECKY2020-097) and individual consent for this retrospective analysis was waived.

Open Access Statement: This is an Open Access article distributed in accordance with the Creative Commons Attribution-NonCommercial-NoDerivs 4.0 International License (CC BY-NC-ND 4.0), which permits the noncommercial replication and distribution of the article with the strict proviso that no changes or edits are made and the original work is properly cited (including links to both the formal publication through the relevant DOI and the license). See: https://creativecommons.org/licenses/by-nc-nd/4.0/.

\section{References}

1. Wells PS, Ginsberg JS, Anderson DR, et al. Use of a clinical model for safe management of patients with suspected pulmonary embolism. Ann Intern Med 1998;129:997-1005.

2. Morella P, Sacco M, Carafa M, et al. Permanent atrial fibrillation and pulmonary embolism in elderly patients without deep vein thrombosis: is there a relationship? Aging Clin Exp Res 2019;31:1121-8.

3. Bikdeli B, Abou Ziki MD, Lip GYH. Pulmonary Embolism and Atrial Fibrillation: Two Sides of the Same Coin? A Systematic Review. Semin Thromb Hemost 2017;43:849-63.

4. Stein PD, Huang H, Afzal A, et al. Incidence of acute pulmonary embolism in a general hospital: relation to age, sex, and race. Chest 1999;116:909-13.

5. Lip GY, Nieuwlaat R, Pisters R, et al. Refining clinical risk stratification for predicting stroke and thromboembolism in atrial fibrillation using a novel risk factor-based approach: the euro heart survey on atrial fibrillation. Chest 2010;137:263-72.

6. Keller K, Hobohm L, Munzel T, et al. Syncope in the German Nationwide inpatient sample - Syncope in atrial fibrillation/flutter is related to pulmonary embolism and is accompanied by higher in-hospital mortality. Eur J Intern 
Med 2019;62:29-36.

7. Kearon C, Akl EA, Ornelas J, et al. Antithrombotic Therapy for VTE Disease: CHEST Guideline and Expert Panel Report. Chest 2016;149:315-52.

8. Chang SS, Dong JZ, Ma CS, et al. Current Status and Time Trends of Oral Anticoagulation Use Among Chinese Patients With Nonvalvular Atrial Fibrillation: The Chinese Atrial Fibrillation Registry Study. Stroke 2016;47:1803-10.

9. Hasanoğlu C, Arguder E, Kilic H, et al. Atrial fibrillation, an obscured cause of pulmonary embolism can be revealed by adding to Wells criteria. J Investig Med 2019;67:1042-7.

10. Ptaszynska-Kopczynska K, Kiluk I, Sobkowicz B. Atrial Fibrillation in Patients with Acute Pulmonary Embolism: Clinical Significance and Impact on Prognosis. Biomed Res Int 2019;2019:7846291.

11. Lutsey PL, Norby FL, Alonso A, et al. Atrial fibrillation and venous thromboembolism: evidence of bidirectionality in the Atherosclerosis Risk in Communities Study. J Thromb Haemost 2018;16:670-9.

12. Wang CC, Lin CL, Wang GJ, et al. Atrial fibrillation

Cite this article as: Bai Y, Yue QM, Sun H, Guo SD, Wang ZZ, Zhong P, Wei XY, Sun L, Liu Y, Shi XB, Liu YT. Prevalence and sex- and age-related risk of pulmonary embolism in in-hospital patients with atrial fibrillation: a multicenter retrospective study from China. Ann Transl Med 2020;8(23):1558. doi: 10.21037/atm-20-2718 associated with increased risk of venous thromboembolism. A population-based cohort study. Thromb Haemost 2015;113:185-92.

13. Basili S, Proietti M, Perticone F, et al. Atrial fibrillation is not associated with increased risk of venous thromboembolism: Results from ARAPACIS study. Thromb Haemost 2015;114:655.

14. Kniffin WD, Jr., Baron JA, Barrett J, et al. The epidemiology of diagnosed pulmonary embolism and deep venous thrombosis in the elderly. Arch Intern Med 1994;154:861-6.

15. Anderson FA Jr, Wheeler HB, Goldberg RJ, et al. A population-based perspective of the hospital incidence and case-fatality rates of deep vein thrombosis and pulmonary embolism. The Worcester DVT Study. Arch Intern Med 1991;151:933-8.

16. Quinn DA, Thompson BT, Terrin ML, et al. A prospective investigation of pulmonary embolism in women and men. JAMA 1992;268:1689-96.

17. Yu QH, Qian HY, Peng HY. [Clinical features of atrial fibrillation complicated with occult pulmonary embolism]. Zhonghua Yi Xue Za Zhi 2019;99:2691-5. 